\title{
AUTOMATED IFC-BASED PROCESSES IN THE CONSTRUCTION SECTOR: A METHOD FOR IMPROVING THE INFORMATION FLOW
}

\author{
Claudio Mirarchi ${ }^{1}$, Daniela Pasini ${ }^{2}$, Alberto Pavan ${ }^{3}$, and Bruno Daniotti ${ }^{4}$
}

\begin{abstract}
One of the main challenges in the implementation of BIM-based processes concerns interoperability issues. In fact, even if IFC format is recognised as an ISO standard, different barriers and problems are often encountered in IFC adoption. Generally, obstacles are due to the difficulty of users in personalising import and export options of IFC formats in BIM authoring tools with the consequent possibility of information loss.

The paper presents a method for improving the information flow, based on the connection of information stored in IFC files and in external databases through automated processes. Therefore, information concerning one single project can be stored in BIModels and linked to external sources or, conversely, referred from external databases to objects in BIModels.

Benefits deriving from the adoption of the proposed solutions concern the limited size of BIModels, the possibility to store information not considered in the IFC schema, and the reduction of IT skills required to building operators for exchanging information in an interoperable way.
\end{abstract}

Keywords: Building Information Modelling, Sharing data, Interoperability, IFC.

\section{INTRODUCTION}

The implementation of BIM-based processes within the building sector requires several efforts for facing issues related to information sharing and exchanging among actors and tools. A BIModel (Succar 2015) is a digital, parametric, intelligent and object-based representation of the characteristics (both physical and functional) of a building. It represents a shared database and knowledge resource for project and building information (National Institute of Building Sciences 2012). Focusing on the concept of shared information among actors, tools and disciplines, open and neutral data schemas were developed to enhance interoperability (Venugopal et al. 2012). Interoperability is considered a key factor in streamlining information flows between different disciplines and influencing the value proposition of BIM in industry. Several solutions have been proposed for ensuring a circular information flow. Among them, the paper focuses on IFCbased solutions (International Organization for Standardization 2013; Pauwels et al. 2017).

Since 1995, BuildingSmart consortium (formerly known as International Alliance for Interoperability until 2008) is working on IFC, a common data model for representing and

1 PhD Candidate, Politecnico di Milano, Department of Architecture, Built environment and Construction engineering, Milano, Italy, claudio.mirarchi@polimi.it

2 PhD Candidate, Politecnico di Milano, Department of Architecture, Built environment and Construction engineering, Milano, Italy, daniela.pasini@polimi.it

3 Research Fellow, Politecnico di Milano, Department of Architecture, Built environment and Construction engineering, Milano, Italy, alberto.pavan@polimi.it

4 Full Professor, Politecnico di Milano, Department of Architecture, Built environment and Construction engineering, Milano, Italy, bruno.daniotti@polimi.it 
describing building processes (Laakso and Kiviniemi 2012; Liebich and Wix 1999). Within this context, BIModels are generally shared through point-to-point exchange of IFC files or through centralised model repositories (Cleveland 2013). Therefore, the importance and usefulness of IFC format is recognised.

However, the correct introduction of digital processes and instruments generally requires specific IT competences of the personnel involved in the field. The lack of automated processes able to support this introduction may hinder the diffusion of correct practices. In fact, the alignment between the competences of the personnel and the requirements of IT is a key aspect for the success of a process of integration of new digital instruments. Therefore, there is a rising need for tools (possibly, automated tools) for simplifying the information flow so that AEC sector can easily adopt IFC within BIMbased processes. Several efforts have been devoted to the development of solutions for sharing information, also relying on semantic web. Due to these issues, the use of IFC is now in changing thanks to the introduction of technologies connected to the semantic web. However, considering that this is still an emerging research area, there is not yet a direct application of these technologies in the construction sector.

\section{RESEARCH METHODOLOGY}

Starting from a review of the state of the art about the use of IFC in the construction sector, the present research defines the main actual issues and some barriers of the processes in action. Considering obstacles of actual processes, the research aims to propose an automated method for improving the workflows of sharing and exchanging information.

Different alternatives have been tested for exchanging information through IFC files. Particularly, commercially available tools have been adopted for translating IFC (BIM authoring tools), for reading IFC (Model viewers) and for writing on IFC (Python-encoded tools).

The proposed method improves the information flow, especially by providing a solution to the issue of information loss, based on the connection of information between native and IFC models using external databases through automated processes.

\section{IFC-BASED INTEROPERABILITY IN THE CONSTRUCTION SECTOR}

The theme of interoperability in the construction sector is of primary interest long since. The well-known characteristics of the sector and of its products have posed several problems in this direction. Therefore, there is the need for solutions for evaluating the correctness and well-formedness of information exchanges through BIModels.

The introduction of BIM-based practices is considered an important mean to improve information management in the construction sector. However, the necessity of different software using proprietary formats may hinder a proper interoperability process. To overcome this problem, IFC has been defined as a standard common language. In fact, IFC schema provides the building blocks for interoperability through its open and neutral data schema (Venugopal et al. 2012). IFC represents geometries, relations, processes and materials, performances, and other properties. However, in several cases, the adoption of IFC is difficult because of IT issues, related to both import and export of IFC files.

Lack of information in sharing BIModels through IFC format is registered in several cases, e.g. when models are exported and imported in the same BIM authoring tool, from architectural models to structural models, from BIM authoring tools to analysis software (e.g. for energy analysis) or from architectural models to FM tools (Hu et al. 2016; Sibenik 
2016). Different reasons have been outlined for this issue. In fact, although IFC is a rich product-modelling schema, it is highly redundant. Moreover, objects, relations, and attributes can be defined in several ways. Thus, data exchanges are often unreliable due to inconsistencies in the different assumptions and interpretations in expressing information (Sacks et al. 2010). Furthermore, instance-level interoperability is not supported (Törmä, 2013). Therefore, IFC implementations need clear guidance for specific purposes and projects (Venugopal et al. 2012).

Generally, the exchange of IFC format does not follow a conversion roundtrip. Therefore, even if models can be exported in IFC (e.g. through BIM authoring tools), the IFC version of the model generally can be used in a read-only mode. Information is lost in a roundtrip and the result is a restructured native model that is generally further developed through extensive manual repairs (Törmä 2013).

Moreover, each individual discipline involves domains that require a very diverse and specific set of information and models to be shared. Therefore, the efficiency of interoperable solutions relies on a flawless and streamlined information flow between different disciplines in a project. However, when IFC files are shared among different tools, lots of criticalities arise, as data can be lost or become inaccurate (Sibenik 2016). Errors generally pertain to syntactic, semantic, and design programming requirements. Among them, syntactic errors are generally caused by the heterogeneous binding structures of BIM authoring tools and the mistaken interpretation of IFC requirements (Lee et al. 2015). When IFC files are imported and exported, problems can be related to the different number of instances, to the failed preservation of Global Unique Identifier or of owner history information, to the loss of data concerning not only geometrical information but also properties of specific entities and their values, to missed information around the inheritance structure or to the unsuccessful transmission of additional properties and requirements (e.g. client-defined or standard-related). Within this context, not only the quality of product models (Solihin et al. 2015), but also the quality of IFC certification process has to be considered (Kiviniemi 2009).

\section{IFC-BASED INFORMATION FLOW}

In order to propose a method for improving interoperable processes, four hypothetical scenarios concerning the implementation of IFC-based information flow are presented:

- Native to IFC, basic process: The BIModel is created using a BIM authoring tool and then exported in IFC, using the standard settings offered by the software and/or off-the-shelf plug-ins.

- Native to IFC, manual integration: The BIModel is created using a BIM authoring tool and then exported in IFC, after setting up and customising the export schema of the software.

- IFC as a native model: The BIModel is created directly in IFC, thus it can be directly read from commercially available software.

- Native to IFC, automated integration: The BIModel is created using a BIM authoring tool and then exported in IFC, using the standard settings offered by the software and integrating the translation with an automated process of information reporting. 


\subsection{Native to IFC, basic process}

Commercially available BIM authoring tools allow users to insert every kind of information in the modelled objects. Considering the uniqueness of the construction products, dedicated information needs to be provided and modelled, according to the requested performances and requirements of products. On the other hand, this hinders the procedure of translation from the native format of BIModels to IFC. In fact, even if several BIM authoring tools allow the export of native formats to IFC, problems can arise when specific property-sets are added by users. Therefore, the translation of these specific property-sets, defined only for a specific project, remains an issue that has to be solved by the final user. In fact, when additional properties are added to a model, some of them can be lost in the translation process using the standard export-settings of commercially available software. Consequently, a lack of information can be registered in the exchanged model with respect to the original one (Figure 1).

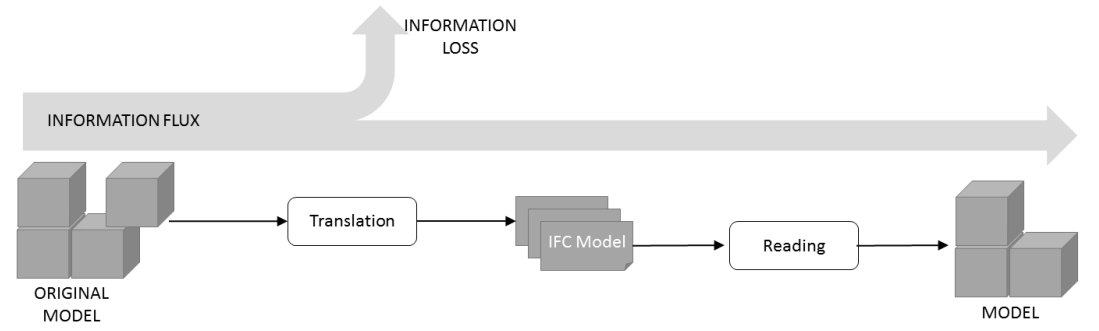

Figure 1: Actual process flow

The abovementioned information flow is generally adopted within the construction sector. Therefore, even if several efforts are devoted by stakeholders in modelling information in an accurate way, a complete information flow is not guaranteed when BIModels are exported and re-imported. In fact, the IFC file obtained in the translation is often incomplete because of a significant information loss, especially of information concerning non-geometrical aspects. Consequently, an external user who has never seen the original model is not able to notice the error, because there are not graphical evidences of the lost information. Therefore, a new kind of interpretation is needed in order to increase and improve the diffusion of effective practices in the information exchange flows.

\subsection{Native to IFC, manual integration}

Several solutions can be adopted to overcome outlined barriers. Among them, users can customise export (and import) options of IFC files, e.g. adapting source code through programming languages (Pasini et al. 2016). Thus, during the translation process, problems are not always connected to the inability of the tools of translating information and storing it in IFC files. Rather, they are mostly related to the restricted expertise of traditional users in the interpretation of the format and in the definition of specific patterns of conversion. In fact, in the construction sector, the average IT competences of users are barely sufficient for a traditional use of standard digital instruments.

The second scenario relies on a customised setting up of the IFC export schema of BIM authoring tools. It allows the creation and translation of a complete IFC model without information loss (Figure 2). 


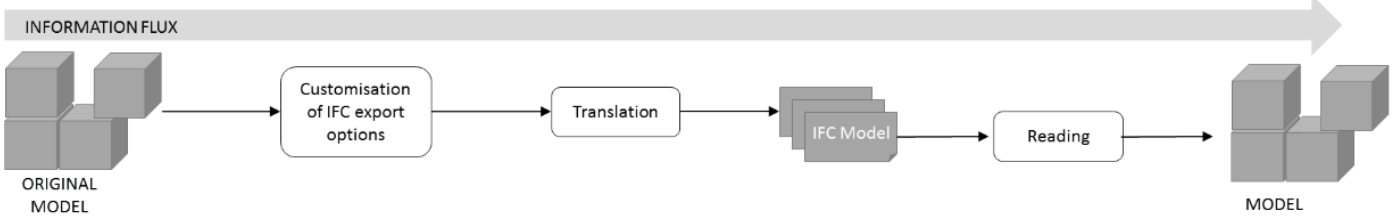

Figure 2: Improved process flow through the customisation of IFC export schema

However, this approach requires a deep knowledge both of the IFC structure and of the export schema adopted by the specific software. Consequently, considering the inadequate IT competencies of users in the construction sector, the presented scenario is rarely applied in a correct way.

\subsection{IFC as a native model}

For avoiding information loss when information is shared and exchanged, it is possible to create BIModels directly in IFC through programming languages. This approach ensures the possibility to model information, especially non-geometrical information, according to the IFC schema. However, it is difficult to produce an entire BIModel by defining the IFC structure based on programming languages.

Based on the mentioned assumption, a scenario has been explored for reporting in the IFC file that part of information that is commonly lost in the roundtrip between proprietary format and IFC format.

As shown in Figure 3, a first step allows the creation of a simplified BIModel directly in IFC, obtaining a native IFC model. When the native IFC model is imported in a BIM authoring tool, no information loss is registered. In a second step, it is possible to write directly in the IFC schema additional information (e.g. information about field management), generally lost in the translation between native and IFC format. In this way, a new BIModel is created; it is characterised by the same geometry of the original model, but it is enriched with more non-geometrical information.

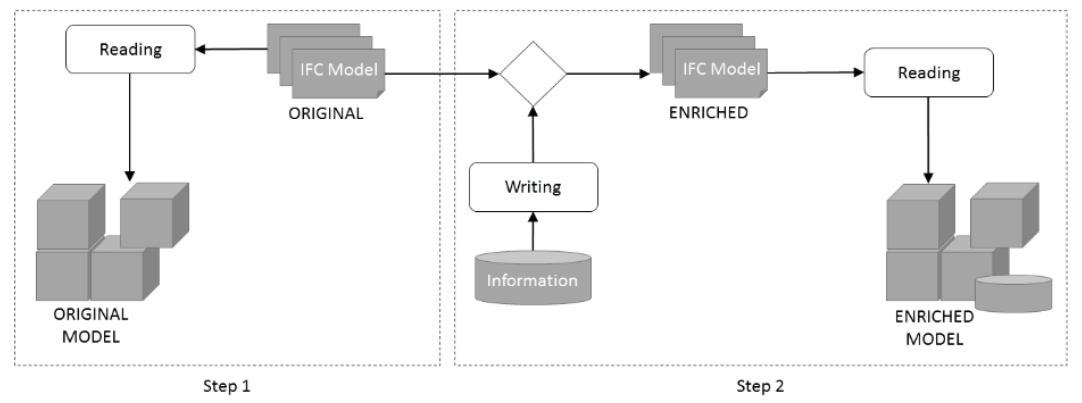

Figure 3: Process flow defined for the sampling test

Therefore, one of the main issue in the data exchange using IFC is represented by the translation process. The presented scenario allows the definition of a BIModel that can be read by different software without information loss. Unfortunately, the construction of a BIModel directly in IFC, even if possible in theory, is in practice inapplicable.

\subsection{Native to IFC, automated integration}

The previous cases show that some of the main issues connected to the information flow can be overcome. However, on the one hand software producers cannot define a priori all the possible information that a user may insert in the model, and, on the other hand, users of the construction sector are not able to effectively act on software settings. Thus, there 
is the need of defining a process that can support the actual information flow (especially for specific property-sets) without requiring specific IT and data management skills to users of BIM authoring tools.

As it is possible to write information directly in an existing IFC file, the last proposed approach considers the possibility to export a BIModel in IFC from a native format and then enrich it with the needed information through an automated process of information transcription. The resulting file would be complete, without information loss (Figure 4).

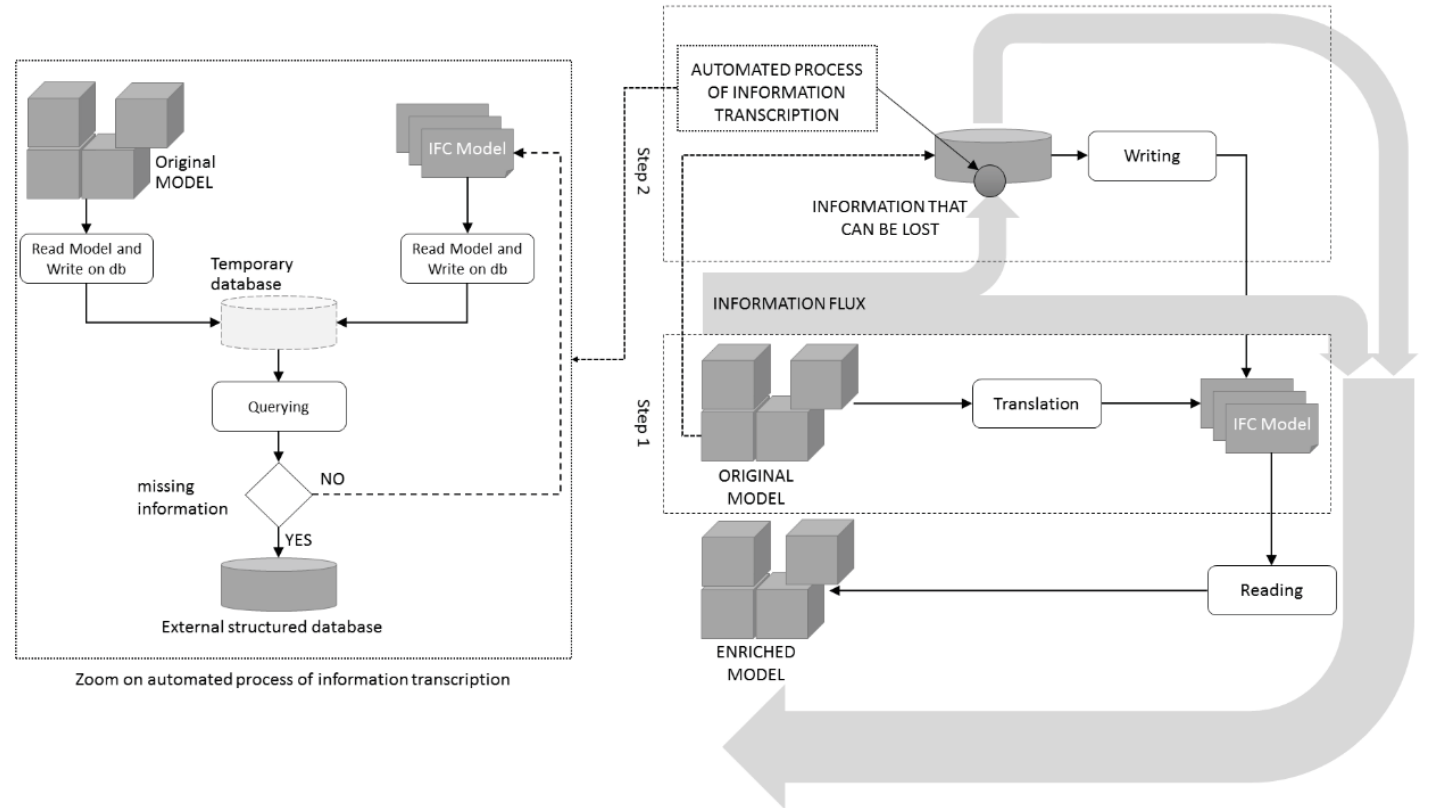

Figure 4: Proposed integrated process

First, the user creates the BIModel, inserting all the relevant information through a BIM authoring tool. In this section, the user complies to the direction imposed by standard and compliant documents defined for the project, without taking into consideration IT issues. Thus, additional fields can be defined for extending information with respect to the standard one proposed by the software and/or commonly defined in IFC. Then, the native BIModel is translated in IFC (Figure 4, Step 1). This phase can be defined directly by the user without acting on the export-settings of commercially available software.

Once the IFC file has been created, information losses can affect the translation process. Therefore, an automated process has been developed in a Python-encoded environment (Figure 4, Step 2). The proposed script (Figure 4, Zoom on automated process of information transcription) reads the information of all the objects both in the native BIModel and in the IFC model, detecting the missing information and writing these ones in a structured database (Figure 5). 


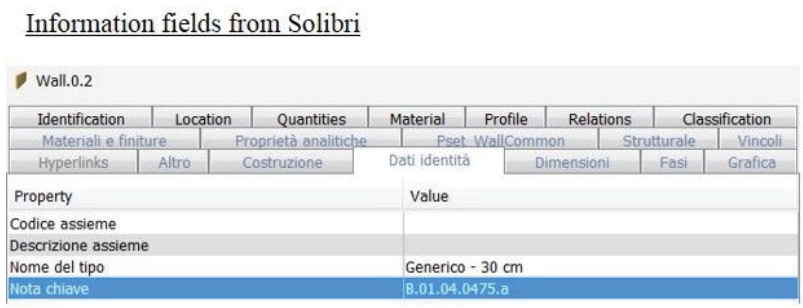

Type information writed in PostgreSQL from IFC

\begin{tabular}{|c|c|c|c|c|c|}
\hline \multicolumn{6}{|c|}{ Output pane } \\
\hline & ta Output & Explain & Messages & History & \\
\hline \multicolumn{5}{|c|}{$\begin{array}{l}\text { nomet } \\
\text { character varvina }\end{array}$} & $\begin{array}{l}\text { notachiave } \\
\text { character varving }\end{array}$ \\
\hline 1 & Muro di & di base: & :Generico & $-30 \mathrm{~cm}: 952$ & B. $01.04 .0475 . \mathrm{a}$ \\
\hline
\end{tabular}

Instance information writed in PostgreSQL from IFC

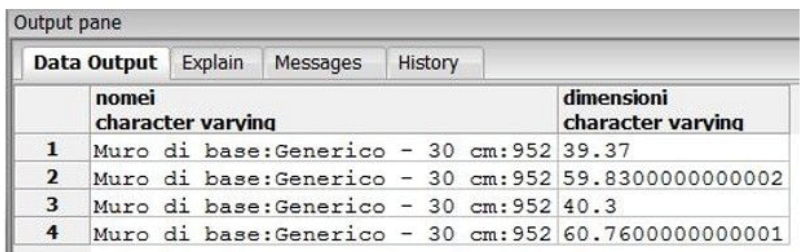

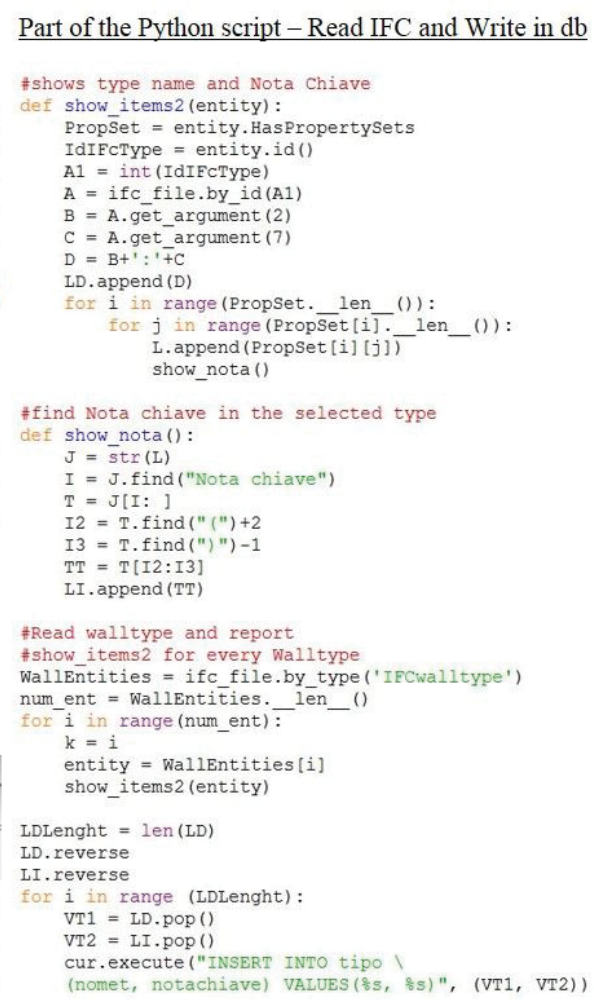

Figure 5: Tests for reading IFC information and writing it in a database

Once the set of information has been written in the database, two different paths can be followed. On the one hand, information can be stored in the database, linked to the model thanks to the unique IDs created by the BIM authoring tool for each modelled object. On the other hand, information can be maintained in the model itself, as shown in Figure 4. This second option can be realised defining a script able to read the information contained in the database and write the information directly in the IFC file, following the schema of the third scenario (in particular Figure 4, Step 2).

Adopting the proposed automated process, the information loss in the roundtrip between native format and IFC format is avoided. Moreover, implementing solutions similar to the developed script in commercially available tools will enhance the information transcription for all the users of the construction sector. Therefore, as specific IT skills are not required, the information flow can improve within the whole supply chain.

\section{CONCLUSIONS}

The paper presents automated IFC-based processes in order to improve interoperability within BIM environments. The main benefits of the proposed solutions concern the limited size of BIModels (as additional information can be stored in external databases, dynamically linked to the BIModel itself), the possibility to store information not considered in the IFC schema, and the limited IT skills required to building operators for exchanging information in an interoperable way. However, tests have been performed focusing on non-geometrical information. Therefore, some issues and difficulties could arise when geometrical parametric information has to be shared.

The test performed during the research shows how the proposed process fits actual practices related to information sharing among users in the construction sector. The issues about information loss are important due to the increasingly value that data are acquiring. 
In this direction, the proposed method is able to guarantee the continuity of the data from the original model, even in the case of users without specific skills in IT and data management areas.

\section{REFERENCES}

Cleveland, A.B. (2013). Interoperability Platform. i-models to Unlock the Value of Information Mobility.

Hu, Z.Z., Zhang, X.Y., Wang, H.W. and Kassem, M. (2016). Improving interoperability between architectural and structural design models: An industry foundation classesbased approach with web-based tools. Autom. Constr. 66, 29-42. doi:10.1016/j.autcon.2016.02.001

International Organization for Standardization. (2013). ISO 16739:2013 - Industry Foundation Classes (IFC) for data sharing in the construction and facility management industries.

Kiviniemi, A. (2009). IFC Certification process and data exchange problems. eWork Ebus. Archit. Eng. Constr. 517-522. doi:10.1201/9780203883327.ch57

Laakso, M. and Kiviniemi, A. (2012). The IFC standard. A review of history, development, and standardization. J. Inf. Technol. Constr. 17, 134-161.

Lee, Y.-C., Eastman, C.M. and Lee, J.-K. (2015). Validations for ensuring the interoperability of data exchange of a building information model. Autom. Constr. 58, 176-195. doi:10.1016/j.autcon.2015.07.010

Liebich, T. and Wix, J. (1999). Highlights of the development process of Industry Foundation Classes, in: Durability of Building Materials and Components.

National Institute of Building Sciences. (2012). National BIM Standard - United States Version 2.

Pasini, D., Ciribini, A.L.C. and Daniotti, B. (2016). Building Information Management for monitoring user behaviour in operational stages, in: BIM Academic Forum, Glasgow.

Pauwels, P., Zhang, S. and Lee, Y.C. (2017). Semantic web technologies in AEC industry: A literature overview. Autom. Constr. 73, 145-165. doi:10.1016/j.autcon.2016.10.003

Sacks, R., Kaner, I., Eastman, C.M. and Jeong, Y.-S. (2010). The Rosewood experiment Building information modeling and interoperability for architectural precast facades. Autom. Constr. 19, 419-432. doi:10.1016/j.autcon.2009.11.012

Sibenik, G. (2016). Building information modelling based interdisciplinary data exchange: a case study, in: BIM Academic Forum, Glasgow.

Solihin, W., Eastman, C. and Lee, Y.C. (2015). Toward robust and quantifiable automated IFC quality validation. Adv. Eng. Informatics 29, 739-756. doi:10.1016/j.aei.2015.07.006

Succar, B. (2015). BIM Dictionary: BIModel. URL http://bimdictionary.com/en/bimodel.

Törmä, S. (2013). Semantic linking of building information models, in: Proceedings of 7th International Conference on Semantic Computing. pp. 412-419. doi:10.1109/ICSC.2013.80

Venugopal, M., Eastman, C.M., Sacks, R. and Teizer, J. (2012). Semantics of model views for information exchanges using the industry foundation class schema. Adv. Eng. Informatics 26, 411-428. doi:10.1016/j.aei.2012.01.005 